\title{
Desarrollo clínico-biológico de ratas alimentadas con dieta semi-purificada a base de clara de huevo
}

\author{
Clinical-biological development of rats fed a semi-purified diet based on egg white
}

\author{
Gabriela González-Infante ${ }^{1}$, Jesús Del Castillo ${ }^{1}$, Francisco Herrera ${ }^{2}$, \\ Guillermina Aguiar², Eunice Marcano², Lusliany Rondón ${ }^{1,3}$
}

\section{Resumen}

\begin{abstract}
Ante la escasez de alimento concentrado para animales en el país, la investigación se centró en elaborar una dieta basada en las directrices del Instituto Americano de Nutrición (AIN) con ingredientes endógenos y probarlos en ratas en gestación, lactancia y crecimiento. En el Experimento 1 se trabajó con 12 ratas Sprague Dawley (SD) machos de 5-6 semanas, la mitad alimentada con dieta control y la otra mitad con dieta no purificada a base de clara de huevo (EWP) durante seis semanas. En el Experimento 2 se trabajó con 10 ratas SD gestantes sometidas a las mismas dietas (control y EWP), para seleccionar 14 crías macho de cuatro semanas de edad para continuar con los mismos regímenes de alimentación por cinco semanas más (7 control y 7 EWP). En ambos experimentos se realizó evaluación de parámetros clínicos-metabólicos (peso corporal, evaluación clínica y estudio en caja metabólica), En el segundo experimento se evaluaron, además, parámetros biológicos (concentración de proteínas y hematología). Los resultados muestran
\end{abstract}

\footnotetext{
${ }^{1}$ Laboratorio de Fisiología Molecular, Centro de Biofísica y Bioquímica, Instituto Venezolano de Investigaciones Científicas (IVIC), Edo. Miranda, Venezuela

${ }^{2}$ Laboratorio de Ecofisiología Vegetal, Centro de Biofísica y Bioquímica, Instituto Venezolano de Investigaciones Científicas (IVIC), Edo. Miranda, Venezuela

${ }^{3}$ E-mail: Luslianyrondonv@hotmail.com
}

Recibido: 14 de enero de 2021

Aceptado para publicación: 22 de julio de 2021

Publicado: 24 de agosto de 2021

CLos autores. Este artículo es publicado por la Rev Inv Vet Perú de la Facultad de Medicina Veterinaria, Universidad Nacional Mayor de San Marcos. Este es un artículo de acceso abierto, distribuido bajo los términos de la licencia Creative Commons Atribución 4.0 Internacional (CC BY 4.0) [https:// creativecommons.org/licenses/by/4.0/deed.es] que permite el uso, distribución y reproducción en cualquier medio, siempre que la obra original sea debidamente citada de su fuente original 
parámetros clínicos-metabólicos estables y parámetros biológicos normales, lo que permite concluir que EWP es adecuada para el desarrollo óptimo y sano de las ratas de laboratorio.

Palabras clave: dieta semi-purificada, proteína de clara de huevo, dieta EWP, Sprague Dawley, parámetros clínicos-metabólicos, niveles de minerales

\section{AbSTRact}

There is a shortage of concentrated feeds for animals in the country and therefore the research focused on developing a diet based on the guidelines of the American Institute of Nutrition (AIN) with endogenous ingredients and testing them in rats in pregnancy, lactation and growth. In Experiment 1, 12 male Sprague Dawley (SD) rats of 56 weeks were worked, half of them fed with a control diet and the other half with a nonpurified diet based on egg white (EWP) for six weeks. In Experiment 2, 10 pregnant SD rats were subjected to the same diets (control and EWP) to select 14 four-week-old male neonates to continue with the same feeding regimens for five more weeks ( 7 in control diet and 7 in EWP). In both experiments, an evaluation of clinical-metabolic parameters (body weight, clinical evaluation and metabolic cage study) was carried out. In the second experiment, biological parameters (protein concentration and hematology) were also evaluated. The results show stable clinical-metabolic parameters and normal biological parameters, which allows to conclude that EWP is suitable for the optimal and healthy development of laboratory rats.

Key words: semi purified diet, egg white protein diet, EWP diet, Sprague Dawley, clinicalmetabolic parameters, biological parameters, mineral levels

\section{INTRODUCCIÓN}

En la actualidad, existe deficiencia en la producción de alimentos para ratas de laboratorio de tipo comercial en Venezuela, lo cual se traduce en una reducción de los estudios médico-farmacéuticos. Ante esto, se decidió elaborar una dieta semi-purificada con base de clara de huevo (Semi-purified egg white protein diet, EWP) para roedores de laboratorio, que satisfaga sus requerimientos nutricionales y les permita un correcto desarrollo. Dicha dieta integró alimentos endógenos que combinados reunieron los requerimientos nutricionales necesarios para el crecimiento y mantenimiento de los roedores, según los lineamientos del Instituto Americano de Nutrición (AIN) para animales de laboratorio (Bieri et al., 1977). En la experimentación animal, el AIN es ampliamente usado como referencia en estudios clínicobiológicos en ratas y ratones, ya que mantiene el crecimiento adecuado de los animales, cumpliendo con sus necesidades energéticas, de macro y micronutrientes (Reeves et al., 1993a).

\section{Materiales y Métodos}

\section{Dieta}

La dieta EWP fue preparada con ingredientes endógenos, bajo las indicaciones reportadas por el AIN, según los requerimientos energéticos para ratas en crecimiento y 
mantenimiento, AIN-93G, AIN-93M (Reeves et al., 1993b) (Cuadro 1). Todos los ingredientes fueron agregados en sus condiciones originales, salvo el maíz y el huevo. El maíz fue procesado en un molino de cuchillas (Fritsch GmbH Pulverisette11) a un tamaño de partículas de $0.5 \mathrm{~mm}$ y luego fue tamizado. La clara de huevo fue deshidratada en estufa Memmert U-40 a $42{ }^{\circ} \mathrm{C}$ durante la noche y pulverizada en una licuadora convencional durante $4 \mathrm{~min}$. Por su parte, las vitaminas (AIN-93-VX) (UPAE-INRA) y los minerales (AIN-93G-MX) (MP Biomedicals), fueron donados al laboratorio. La composición de ambas mezclas se halla publicada en el trabajo de Reeves et al. (1993b). La harina de arroz, el aceite de maíz y el papelón negro (también conocido como dark brown sugar, panela, piloncillo o chancaca) fueron obtenidos de manera comercial. Los ingredientes fueron mezclados mecánicamente durante $15 \mathrm{~min}$ en un procesador de uso doméstico. Se realizó análisis proximal y mineral de las dietas (Cuadros 2 y 3 ).

\section{Análisis Proximal y de Minerales}

La composición química se determinó según los métodos estándares indicados por la Asociación Oficial de Químicos Agrícolas (A.O.A.C.) (Horwitz y Latimer, 2005). El contenido de carbohidratos totales fue calculado por diferencia en base seca. El contenido mineral en las muestras se determinó según métodos descritos en la literatura (Windsor y Denton, 1978; Olivares et al., 2016).

\section{Animales}

Las ratas se obtuvieron del Bioterio Central del Instituto Venezolano de Investigaciones Científicas (IVIC). Se mantuvieron con agua y comida ad libitum hasta el día de su sacrificio y se alojaron en salas con temperatura controlada $\left(22^{\circ} \mathrm{C}\right)$ con un ciclo de luz-oscuridad de 12:12h. Las ratas fueron manipuladas de acuerdo con las recomendaciones del Comité de Bioética del IVIC y la
Directiva de la CE 86/609/CEE para experimentos con animales.

\section{Experimento 1}

Se trabajó con 12 ratas macho de la cepa Sprague Dawley (SD), los cuales fueron alimentados desde su destete (cuarta semana de vida), con dieta control (dieta comercial estándar para roedores: Protinal ${ }^{\circledR}$ ). En el transcurso de la semana cinco a seis de vida, las ratas fueron distribuidas de manera aleatoria en dos grupos: seis alimentadas con dieta control y seis con dieta EWP, esta última preparada en el Laboratorio de Fisiología Molecular del Centro de Biofísica y Bioquímica del IVIC. Ambos grupos se mantuvieron bajo tratamiento durante seis semanas. Se registró el peso corporal (PC) cada dos días y la evaluación clínica diariamente, agrupando los datos por semana de tratamiento. En la semana 4 del estudio, las ratas se sometieron a caja metabólica durante 48 horas (Figura 1) para la obtención de muestras de orina y heces. Las ratas fueron sacrificadas en la semana 6 mediante extracción total de sangre por punción cardíaca bajo anestesia con Tiopental sódico $(20-25 \mathrm{mg} / \mathrm{kg})$, por vía intraperitoneal. La sangre fue recolectada en tubos heparinizados y centrifugados a $3500 \mathrm{~g}$ durante $10 \mathrm{~min}$ a $4{ }^{\circ} \mathrm{C}$. El plasma se separó y almacenó a $-20^{\circ} \mathrm{C}$ hasta su análisis.

\section{Experimento 2}

En esta fase, las ratas (machos y hembras SD) fueron seleccionadas para apareamiento según edad y condición clínica. Se realizó un apareamiento monogámico por siete días, durante el cual se alimentaron con dieta control o EWP según el grupo experimental. En el día 8 los machos fueron retirados y las hembras fueron alojadas en cajas individuales de plexiglass, para continuar con su posible gestación en ambiente controlado con la misma dieta que consumieron durante el periodo de monta. Se registró el peso de las hembras cada dos días, considerándose como gestantes aquellas que tuvieron mayor aumento de peso corporal. 
Cuadro 1. Composición de las dietas experimentales

\begin{tabular}{|c|c|c|c|c|}
\hline Ingredientes & AIN-93G ${ }^{1}$ & AIN-93M ${ }^{2}$ & Ingredientes & $\mathrm{EWP}^{3}$ \\
\hline Caseína & 200 & 140 & Clara de huevo & 170 \\
\hline Maicena & 397.5 & 465.7 & Maíz amarillo & 300 \\
\hline Fécula de maíz & 132 & 155 & Harina de arroz & 289 \\
\hline Sacarosa & 100 & 100 & Papelón negro & 150 \\
\hline $\begin{array}{l}\text { Aceite de soja (sin } \\
\text { aditivos) }\end{array}$ & 70 & 40 & Aceite de maíz & 45 \\
\hline $\begin{array}{l}\text { Mezcla de minerales } \\
\text { (AIN-93M-MX) }\end{array}$ & 35 & 35 & $\begin{array}{l}\text { Mezcla de } \\
\text { minerales (AIN- } \\
\text { 93G-MX) }\end{array}$ & 35 \\
\hline $\begin{array}{l}\text { Mezcla de vitaminas } \\
\text { (AIN-93-VX) }\end{array}$ & 10 & 10 & $\begin{array}{l}\text { Mezcla de } \\
\text { vitaminas (AIN- } \\
\text { 93-VX) }\end{array}$ & 10 \\
\hline $\begin{array}{l}\text { Bitartrato de colina } \\
(41.1 \% \text { de colina) }\end{array}$ & 2.5 & 2.5 & Acetil colina & 1 \\
\hline Fibra & 50 & 50 & Folato & 0.0000452 \\
\hline L-cistina & 3 & 1.8 & & \\
\hline $\begin{array}{l}\text { Terc- } \\
\text { butilhidroquinona }\end{array}$ & 0.014 & 0.008 & & \\
\hline
\end{tabular}

${ }^{1}$ Dieta AIN para roedores en crecimiento y gestantes

2 Dieta AIN para roedores adultos

${ }^{3}$ Dieta semi-purificada con base de clara de huevo

Los valores son expresados en gramos por kilogramo de dieta $(\mathrm{g} / \mathrm{kg})$

Se trabajó con 10 ratas SD gestantes (14 semanas de edad y 1 semana de gestación). Los animales fueron alimentados durante su gestación y lactancia con dieta control $(n=5)$ y dieta EWP $(n=5)$. Las crías fueron pesadas al nacimiento, $\mathrm{y}$ fueron destetadas a las cuatro semanas de vida. Al destete, se seleccionaron siete ratas por grupo de alimentación (dieta control y dieta EWP). Ambos grupos se mantuvieron bajo el mismo tratamiento original durante seis semanas. El registro de peso, evaluación clínica y toma de muestras de heces y orina y sacrificio se hizo como se indica en el Experimento 1 (Figura 2).

\section{Parámetros Clínico-Metabólicos}

- Peso corporal. Los animales fueron pesados de manera individual e interdiaria, empleando una balanza electrónica Körper EK3651.
- Evaluación clínica. Se realizó revisión directa diaria de signos físico-clínicos, llenando un instrumento de observación descrito por Morton y Griffiths (1985). Los resultados fueron analizados mediante la prueba de Kolmogorov-Smirnov.

- Motricidad. Los animales fueron colocados en una caja de plexiglás transparente y grabados por medio de un programa de seguimiento y cuantificación de pasos «Video Tracker Software» (OSP, Física de Código Abierto) (VT) (Endo et al., 2016). Se estandarizó el tiempo exploración, el cual osciló entre 40 segundos y 2 minutos. A partir de allí, se cuantificó el número de movimientos ejecutados por cada rata en 2 minutos contados desde su ingreso a la caja. Los datos numéricos arrojados por VT fueron graficados en los programas Microsoft Office Excel o Graphpad y 
Cuadro 2. Análisis proximal de dietas AIN-93G, AIN-93M, control y EWP

\begin{tabular}{lcccc}
\hline Parámetro & AIN-93G & AIN-93M & Control & EWP $^{3}$ \\
\hline Humedad $(\mathrm{g} .100 \mathrm{~g})$ & 6.6 & 6.8 & 7.2 & 6.5 \\
Cenizas $(\mathrm{g} .100 \mathrm{~g})$ & 4.2 & 3.9 & 7.2 & 4.2 \\
Grasas $(\mathrm{g} .100 \mathrm{~g})$ & 7.0 & 4.0 & 4.1 & 5.8 \\
Proteínas $(\mathrm{g} .100 \mathrm{~g})$ & 17.9 & 12.6 & 37.2 & 17.2 \\
Carbohidratos totales $(\mathrm{g} .100 \mathrm{~g})$ & 64.4 & 72.7 & 44.4 & 66.4 \\
Calorías (kcal) & 376.6 & 360.1 & 309.1 & 377.1 \\
Fibra (\%) & 5.0 & 5.0 & 13.51 & 2.25 \\
\hline
\end{tabular}

${ }^{1}$ Dieta AIN para roedores en crecimiento y gestantes

2 Dieta AIN para roedores adultos

${ }^{3}$ Dieta semi-purificada con base de clara de huevo

Cuadro 3. Contenido de minerales ( $\mathrm{mg} / \mathrm{kg}$ ) en dietas AIN-93G, AIN-93M, control y EWP

\begin{tabular}{lcccc}
\hline Mineral & AIN-93G $^{1}$ & AIN-93M $^{2}$ & Control & EWP $^{3}$ \\
\hline Aluminio (Al) & & & $130 \pm 26$ & $86 \pm 1$ \\
Bario (Ba) & & & $16,2 \pm 0,5$ & $4,6 \pm 0,1$ \\
Hierro (Fe) & 45 & 45 & $667 \pm 102$ & $348 \pm 35$ \\
Cobre (Cu) & 6 & 6 & $49 \pm 1$ & $17 \pm 2$ \\
Manganeso (Mn) & 10 & 10 & $86 \pm 2$ & $51 \pm 1$ \\
Estroncio (Sr) & & & $29,5 \pm 0,2$ & $18,8 \pm 0,3$ \\
Zinc (Zn) & 38 & 35 & $575 \pm 23$ & $73 \pm 1$ \\
Calcio (Ca) & 5.000 & 5.000 & $4.493 \pm 72$ & $3.610 \pm 788$ \\
Potasio (K) & 3.600 & 3.600 & $5.906 \pm 6$ & $5.145 \pm 546$ \\
Magnesio (Mg) & 513 & 511 & $989 \pm 72$ & $723 \pm 207$ \\
Sodio (Na) & 1.039 & 1.033 & $1.105 \pm 64$ & $2.547 \pm 449$ \\
Fósforo (P) & 3.000 & 3.000 & $2.667 \pm 297$ & $2.105 \pm 447$ \\
\hline
\end{tabular}

${ }^{1}$ Dieta AIN para roedores en crecimiento y gestantes

2 Dieta AIN para roedores adultos

${ }^{3}$ Dieta semi-purificada con base de clara de huevo

comparados con los gráficos arrojados por VT. Se estableció una comparación entre la cantidad de movimientos de las ratas y sus controles.

- Caja metabólica. Para cuantificar la ingesta de agua y alimento, la excreción de orina y heces, y observar el compor- tamiento aislado de cada rata, los grupos fueron colocados individualmente en una caja metabólica estándar para ratas (Tecniplast, USA) durante 48h. El agua y el alimento fue suministrado ad libitum y la orina y heces fue recolectada cada $24 \mathrm{~h}$ en recipientes volumétricos calibra- 


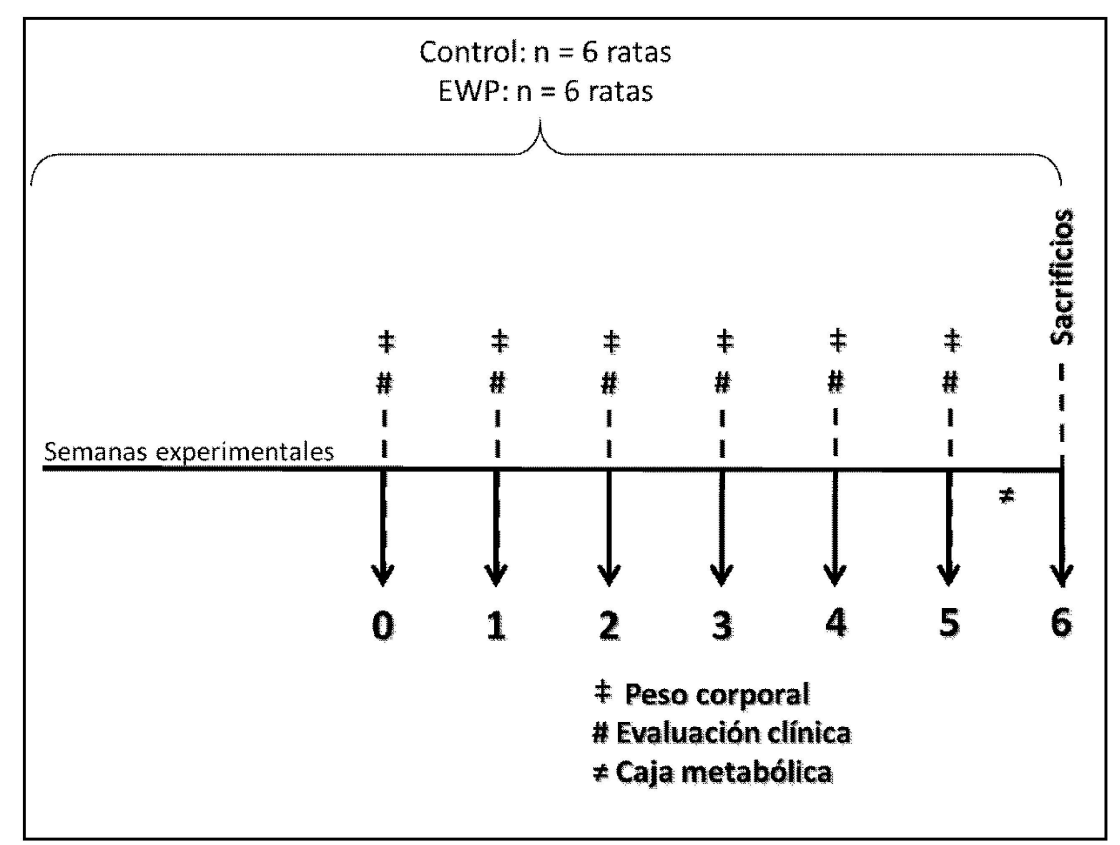

Figura 1. Diseño del Experimento 1

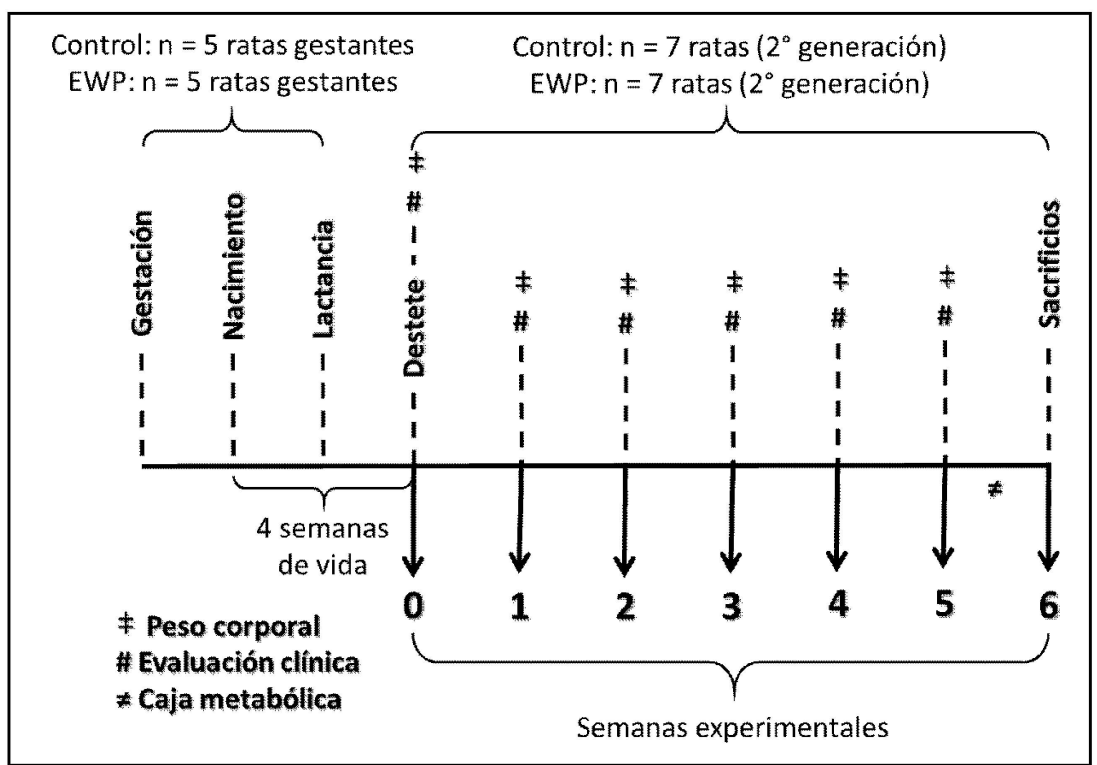

Figura 2. Diseño del Experimento 2 


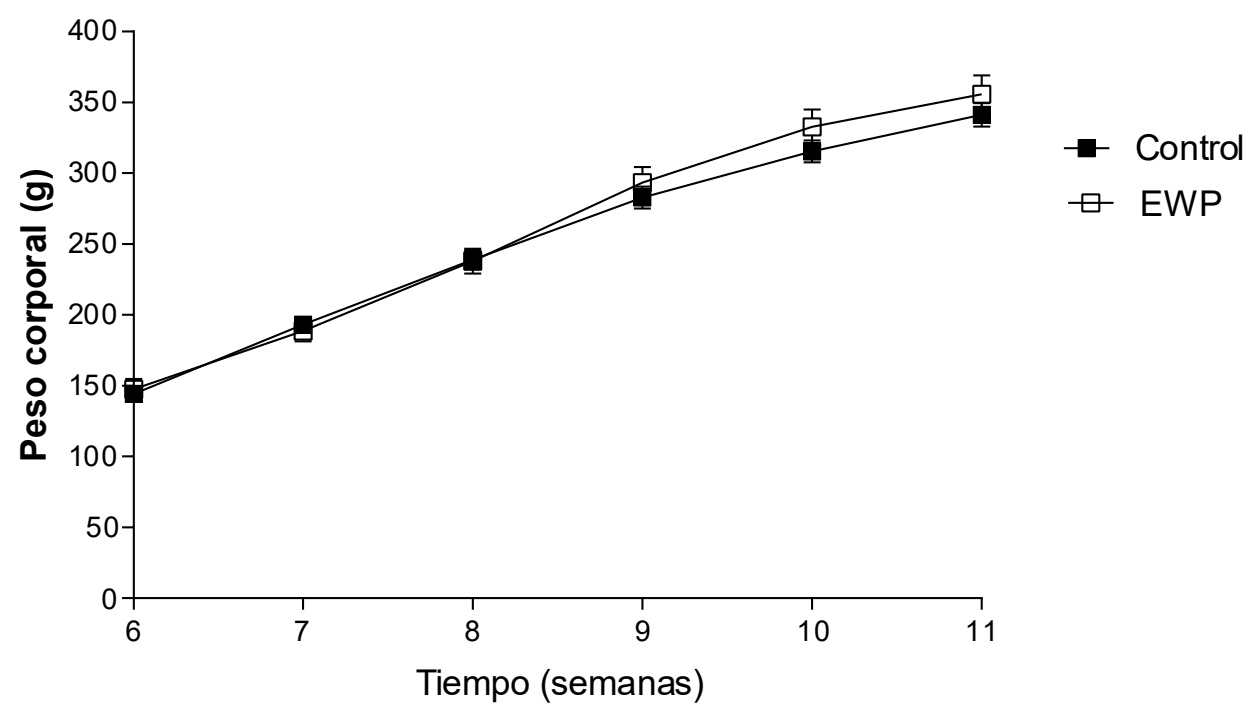

Figura 3. Evolución del peso corporal en ratas Sprague Dawley alimentadas con dieta control o EWP (semi-purificada con base de clara de huevo). Los valores se expresan como medias \pm SEM en gramos ( $n=6$ por grupo)

dos (Tecniplast, USA). Las heces fueron pesadas y sometidas a deshidratación por $24 \mathrm{~h}$ a $50{ }^{\circ} \mathrm{C}$ en una estufa Heratherm 51028123 (ThermoFisher). El balance hídrico se calculó restando el total de egresos (orina + heces) al total de ingresos (agua consumida) y dividido entre el peso corporal, (Saínz, 2005; Cheuvront et al., 2011; Rieble y Davy, 2013). El porcentaje de excreción se calculó tomando como punto de inicio el agua neta consumida (100\%) y restando el egreso como porcentaje excretado. Estas estimaciones no incluyen pérdidas de agua de otro tipo.

\section{Parámetros Biológicos}

Se determinó la concentración de proteínas en plasma y orina con un espectrofotómetro Spectronic 1201 (Milton Roy) mediante la técnica de Bradford (Bradford, 1976). El hemograma fue determinado mediante métodos convencionales (Blumenreich,
1990), con el analizador hematológico de micro-muestreo ABX MICROS ES60. Se determinaron los valores de leucocitos (WBC), hemoglobina (HGB), eritrocitos (RBC), hematocrito (HCT) volumen corpuscular medio (MCV), hemoglobina corpuscular media $(\mathrm{MCH})$ y concentración de hemoglobina corpuscular media (MCHC), así como el tamaño y dispersión de los eritrocitos (RDW-CV, RDW-SD), el conteo de plaquetas (PLT), volumen plaquetario medio (MPV), índice de distribución de plaquetas (PDW), porcentaje de procalcitonina (PCT), y concentración de glucosa (GLU), colesterol (COL), triglicéridos (TRI) y lipoproteínas de muy baja densidad (VLDL).

\section{Análisis Estadístico}

Los valores son expresados como media \pm SEM. Las diferencias entre los grupos experimentales fueron determinadas mediante análisis de varianza de dos vías seguido del 
Cuadro 4. Evaluación clínica de ratas Sprague Dawley alimentadas con dieta control o dieta EWP (semi-purificada con base de clara de huevo) durante seis semanas a partir del destete

\begin{tabular}{lcc}
\hline Condición & Control & EWP \\
\hline Normal (0-6) & $6 / 6$ & $6 / 6$ \\
Monitoreo continuo (7-12) & $0 / 6$ & $0 / 6$ \\
Modificar tratamiento (13-18) & $0 / 6$ & $0 / 6$ \\
Detener experimento (19-25) & $0 / 6$ & $0 / 6$ \\
Motricidad (cm/s) & $0.013 \pm 0.002$ & $0.015 \pm 0.001$ \\
\hline
\end{tabular}

Los valores se expresan como número de animales respondedores/número de ratas en el grupo estudiado. Análisis estadístico realizado por Kolmogorov-Smirnov

Parámetros como respiración, micción, sudoración o suavidad del pelaje, entre otros, se categorizan como "normal" para continuar el experimento, "monitoreo continuo" para observar con mayor detalle al grupo o individuo, "modificar tratamiento" para continuar con el suministro de la dieta, o "detener el tratamiento" para evitar el sufrimiento y malestar de los animales (Morton y Griffiths, 1985).

ensayo de Tukey. Se uso el análisis de Kolmogorov-Smirnov en datos cualitativos. Las diferencias fueron consideradas significativas a partir de $\mathrm{p}<0.05$. Se utilizó Graphpad Prism (Systat Software Inc., USA) como programa para análisis de datos.

\section{Resultados y Discusión}

\section{Experimento 1}

Conforme a lo observado en investigaciones de AIN con dietas purificadas. (Reeves et al., 1993a), en este estudio no hubo diferencias en PC entre las ratas alimentadas con dieta control y con dieta EWP entre el destete hasta las 11-12 semanas de vida $(355.75 \pm 13.39 \mathrm{~g}$ y $341.17 \pm 8.24 \mathrm{~g}$, respectivamente) (Figura 3).

Tal como se esperaba, ambos grupos mostraron un comportamiento clínico normal, libre de patologías o afecciones en apariencia y fisiología al alimentarse con la dieta EWP (Semon et al., 1987) (Cuadro 4). La literatu- ra ha demostrado la utilidad de cuantificar el movimiento espontáneo en los roedores, bien sea mediante la codificación ciega de expresiones faciales o sobre el tiempo empleado en explorar su entorno, como parte de una evaluación físico-clínica completa (Sotocinal et al., 2011). El presente estudio confirma el bienestar de los animales en motricidad y comportamiento general, según lo esperado en este tipo de investigaciones (Morton y Griffiths, 1985; Endo et al., 2016).

En el Cuadro 5 se presentan los resultados de la caja metabólica. A pesar de que el contenido de Na en dieta EWP $(0.23 \% \mathrm{Na}$ $\mathrm{p} / \mathrm{p}$, normosódica [Martus et al., 2005]) es ligeramente mayor que en el grupo control, el grupo EWP mostró una menor ingesta de alimentos comparado (Control: $21.95 \pm 0.38 \mathrm{~g}$; EWP: $18.63 \pm 0.79 \mathrm{~g}$ ) y, por consiguiente, un menor peso de heces $(3.75 \pm 0.13 \mathrm{~g}$ y $1.08 \pm$ $0.07 \mathrm{~g}$, respectivamente). Diversos factores pueden estar asociados al menor consumo de alimento como sabor y textura del alimento (Naim et al., 1986) o contenido de $\mathrm{Na}$ (Rondón et al., 2014). Sin embargo, la re- 
Cuadro 5. Ingesta de alimento y agua, excreción de orina, contenido de agua en heces y peso seco de heces en ratas Sprague Dawley alimentadas con dieta control y EWP (semi-purificada con base de clara de huevo)

\begin{tabular}{lcc}
\hline Parámetro & Control & EWP \\
\hline Ingesta de alimento $(\mathrm{g} / 24 \mathrm{~h})$ & $21.95 \pm 0.38$ & $18.63 \pm 0.79^{* *}$ \\
Ingesta de agua $(\mathrm{ml} / 24 \mathrm{~h})$ & $34.25 \pm 1.07$ & $26.77 \pm 2.85^{*}$ \\
Excreción de orina $(\mathrm{ml} / 24 \mathrm{~h})$ & $17.67 \pm 0.71$ & $17.33 \pm 2.15$ \\
Contenido de agua fecal $(\%)$ & $56.35 \pm 2.64$ & $56.67 \pm 2.60$ \\
Peso de heces secas $(\mathrm{g})$ & $3.75 \pm 0.13$ & $1.08 \pm 0.07^{* * *}$ \\
Balance hídrico $(\mathrm{ml})$ & $0.035 \pm 0.003$ & $0.023 \pm 0.002^{* *}$ \\
Excreción de agua $(\%)$ & $67.4 \pm 1.71$ & $70.6 \pm 2.23$ \\
\hline
\end{tabular}

Los valores se expresan como media \pm SEM $(n=6)$

El análisis estadístico se realizó mediante la prueba $t$ de Student para datos no apareados seguida de la prueba F para comparar las varianzas. ${ }^{* * *} \mathrm{p}<0.001,{ }^{* *} \mathrm{p}<0.01, * \mathrm{p}<0.05$

Balance hídrico $(\mathrm{ml})=($ Ingreso $($ ingesta de agua) - Egreso $($ orina + heces $)) / P C$

Balance hídrico $(\%)=($ Egreso $(\mathrm{ml}) * 100) /$ Ingreso $(\mathrm{ml})$

ducción en la ingesta no afectó la ganancia de peso ni el estado clínico de las ratas. Asimismo, el balance hídrico corregido por PC indicó un balance significativamente más bajo en ratas alimentadas con dieta EWP (-0.012 \pm 0.001 comparado con su control); sin embargo, este dato se correlaciona correctamente con el ingreso y el egreso de agua (Cheuvront et al., 2011). Por otra parte, el porcentaje de excreción de agua demostró que no hubo diferencia significativa entre grupos $(67.4 \pm 1.71 \%$ en grupo control y $70.6 \pm$ $2.23 \%$ para EWP). Esto se asocia con una funcionalidad correcta de los riñones por equilibrio hidroelectrolítico de los fluidos corporales (Roumelioti et al., 2018).

\section{Experimento 2}

En la Figura 4 se observa el PC y evolución del PC en ratas SD alimentadas con las dietas en las fases de neonatos, lactancia y destete-crecimiento. El PC de neonatos al nacimiento fue mayor en el grupo de madres alimentadas con EWP $(+1.56 \pm 0.18 \mathrm{~g})$ (Fi- gura 4A), resultado que puede deberse al contenido de grasas y carbohidratos totales en la dieta EWP (Ramirez y Friedman, 1990). A pesar de ello, a los tres días de nacidos igualaron el PC con el grupo alimentado con la dieta control, siendo similar durante toda la lactancia (27 días) (Figura 4 B), así como durante la fase de destete-crecimiento ( $\mathrm{Fi}$ gura 4C), excepto en la semana 5 de vida, donde la ganancia de peso fue mayor en el grupo EW, posiblemente debido al proceso de adaptación al cambio de alimento en el destete (Levin y Keesey, 1998).

Tal como se observó en el Experimento 1, ambos grupos presentaron signos físicoclínicos y motricidad similares, así como un comportamiento semejante sin cambios anormales (Cuadro 6).

$\mathrm{Al}$ igual que en Experimento 1, las ratas alimentadas con dieta EWP mostraron una menor ingesta de alimento y, por ende, un menor peso de heces secas que en el grupo 


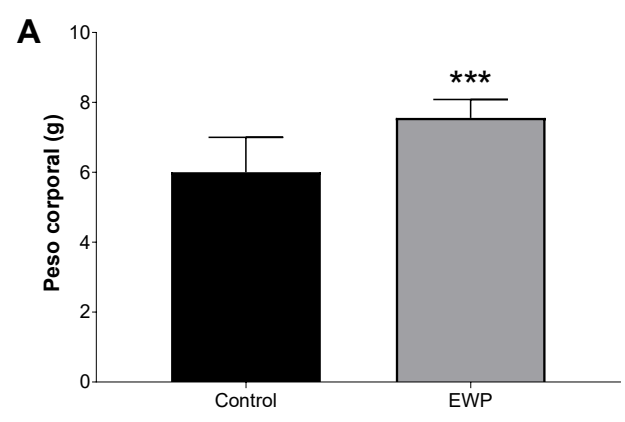

B

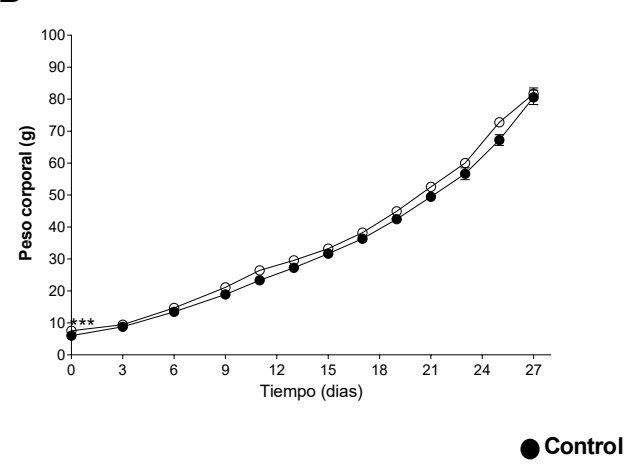

C

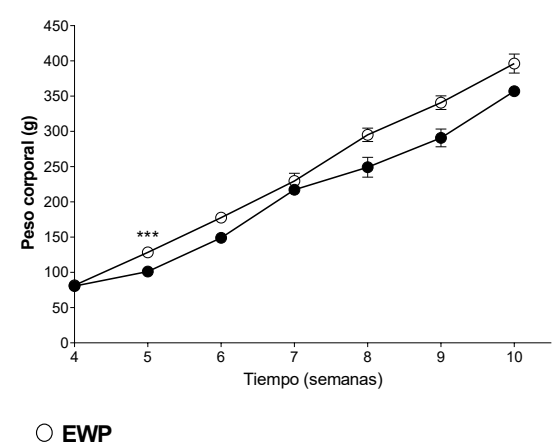

Figura 4. Evolución del peso corporal en ratas Sprague Dawley alimentadas con dieta control o dieta EWP (semi-purificada con base de clara de huevo). (A) Recién nacido; (B) Lactancia; (C) Destete y crecimiento. Los valores se expresan como medias $\pm \mathrm{SEM}$ en gramos ( $\mathrm{n}=6$ por grupo). El análisis estadístico se realizó utilizando $t$ student seguido por el método de corrección para múltiples comparaciones de Holm-Sidak. *** $\mathrm{p}<0.001$

alimentado con dieta control $(-4.36 \pm 0.53,-$ $5.71 \pm 0.27$, respectivamente) (Cuadro 7). A pesar de que se encuentra en un rango de normalidad (Rieble y Davy, 2013), el balance hídrico corregido por peso corporal es significativamente menor en el grupo EWP que en el grupo control $(-0.019 \pm 0.001)$ tendiendo ambos a 0 , indicando un balance hidroelectrolítico adecuado. No obstante, contrario a lo observado en el Experimento 1, el porcentaje de excreción de agua fue significativamente superior en el grupo EWP comparado al grupo control $(+9.76 \pm 1.13)$ (Cuadro 7).
Por otra parte, a pesar de que la excreción de agua y el balance hídrico en el Experimento 2 fueron diferentes entre sí, los valores se encuentran dentro de los rangos de normalidad establecidos (Rieble y Davy, 2013). No obstante, el tiempo de experimentación en esta fase del estudio es más prolongada que el Experimento 1, abarcando desde la gestación y lactancia, hasta la semana 11 del experimento; lo que podría llevar a cambios metabólicos-fisiológicos importantes. (Kind et al., 2006. Cristians et al., 2019). A su vez, se debe considerar que la dieta EWP posee menor cantidad de potasio (K) y fibra que la dieta control, generando un 
Cuadro 6. Evaluación clínica de ratas Sprague Dawley alimentadas con dieta control o dieta EWP (semi-purificada con base de clara de huevo) durante seis semanas a partir del destete

\begin{tabular}{lcc}
\hline Condición & Control & EWP \\
\hline Normal (0-6) & $8 / 8$ & $5 / 5$ \\
Monitoreo continuo (7-12) & $0 / 8$ & $0 / 5$ \\
Modificar tratamiento (13-18) & $0 / 8$ & $0 / 5$ \\
Detener experimento (19-25) & $0 / 8$ & $0 / 5$ \\
\hline
\end{tabular}

Los valores se expresan como número de animales respondedores/número de ratas en el grupo estudiado. Análisis estadístico realizado por Kolmogorov-Smirnov

Parámetros como respiración, micción, sudoración o suavidad del pelaje, entre otros, se categorizan como "normal" para continuar el experimento, "monitoreo continuo" para observar con mayor detalle al grupo o individuo, "modificar tratamiento" para continuar con el suministro de la dieta, o "detener el tratamiento" para evitar el sufrimiento y malestar de los animales (Morton y Griffiths, 1985).

Cuadro 7. Ingesta de alimento y agua, excreción de orina, contenido de agua en heces y peso seco de heces en ratas Sprague Dawley alimentadas con dieta control y EWP (semi-purificada con base de clara de huevo)

\begin{tabular}{lcc}
\hline Parámetro & Control & EWP \\
\hline Ingesta de alimento $(\mathrm{g} / 24 \mathrm{~h})$ & $23.50 \pm 0.77(7)$ & $19.14 \pm 1.30(5)^{*}$ \\
Ingesta de agua $(\mathrm{ml} / 24 \mathrm{~h})$ & $36.64 \pm 0.88(7)$ & $29.03 \pm 3.04(5)^{*}$ \\
Excreción de orina $(\mathrm{ml} / 24 \mathrm{~h})$ & $17.50 \pm 1.15(7)$ & $19.00 \pm 2.63(5)$ \\
Contenido de agua fecal $(\%)$ & $51.78 \pm 5.35(7)$ & $54.16 \pm 3.56(5)$ \\
Peso de heces secas $(\mathrm{g})$ & $8.80 \pm 0.50(7)$ & $3.09 \pm 0.27(5)^{* * *}$ \\
Balance hídrico $(\mathrm{ml})$ & $0.042 \pm 0.003(7)$ & $0.023 \pm 0.004(5)^{* * *}$ \\
Excreción de agua $(\%)$ & $60.12 \pm 2.65(7)$ & $69.88 \pm 3.78(5)^{*}$ \\
$\begin{array}{l}\text { Excreción urinaria de proteínas } \\
\text { (mg/24h) }\end{array}$ & $38.49 \pm 2.58(7)$ & $32.94 \pm 11.76(5)$ \\
$\begin{array}{l}\text { Concentración plasmática de } \\
\text { proteínas(mg/ml) }\end{array}$ & $62.42 \pm 5.58(5)$ & $59.23 \pm 9.57(3)$ \\
\hline
\end{tabular}

Los valores se expresan como media \pm SEM (número de ratas entre paréntesis)

El análisis estadístico se realizó mediante la prueba $t$ de Student para datos no apareados seguida de la prueba $\mathrm{F}$ para comparar las varianzas. ${ }^{* * *} \mathrm{p}<0.001, * * \mathrm{p}<0.01,{ }^{*} \mathrm{p}<0.05$

Balance hídrico $(\mathrm{ml})=($ Ingreso (ingesta de agua) - Egreso (orina + heces) $) / P C$

Balance hídrico $(\%)=($ Egreso $(\mathrm{ml}) * 100) /$ Ingreso $(\mathrm{ml})$ 
Cuadro 8. Análisis bioquímico de muestras de sangre en ratas Sprague Dawley alimentadas con dieta control y dieta EWP (semi-purificada con base de clara de huevo)

\begin{tabular}{lcc}
\hline Parámetro & Control & EWP \\
\hline WBC $(\mu \mathrm{m})$ & $5.800 \pm 2.150(3)$ & $7.100 \pm 793.7(3)$ \\
HGB $(\mathrm{g} / \mathrm{dl})$ & $14.83 \pm 0.03(3)$ & $15.43 \pm 0.72(3)$ \\
RBC $(\mu \mathrm{m})$ & $6.496 .667 \pm 46.667(3)$ & $6.840 .000 \pm 330.051(3)$ \\
HCT $(\%)$ & $43.43 \pm 0.35(3)$ & $43.47 \pm 2.24(3)$ \\
MCV $(\mathrm{fl})$ & $66.90 \pm 0.85(3)$ & $63.60 \pm 0.23(3)$ \\
MCH $(\mathrm{pg})$ & $22.77 \pm 0.12(3)$ & $22.50 \pm 0.15(3)$ \\
MCHC $(\mathrm{g} / \mathrm{dl})$ & $34.13 \pm 0.35(3)$ & $35.47 \pm 0.33(3)$ \\
RDW-CV $(\%)$ & $13.50 \pm 0.29(3)$ & $14.27 \pm 0.13(3)$ \\
RDW-SD $(\mathrm{fl})$ & $25.63 \pm 0.43(3)$ & $25.60 \pm 0.00(3)$ \\
PLT $(\mu \mathrm{l})$ & $586.000 \pm 19.140(3)$ & $697.667 \pm 43.529(3)$ \\
MPV $(\mathrm{fl})$ & $9.13 \pm 0.19(3)$ & $9.77 \pm 0.09(3)^{*}$ \\
PDW $(\%)$ & $14.40 \pm 0.15(3)$ & $14.77 \pm 0.03(3)$ \\
PCT $(\%)$ & $0.66 \pm 0.15(3)$ & $0.65 \pm 0.02(2)$ \\
GLU $(\mathrm{mg} / \mathrm{dl})$ & $168.70 \pm 10.17(3)$ & $179.00 \pm 10.07(3)$ \\
COL $(\mathrm{mg} / \mathrm{dl})$ & $37.67 \pm 2.60(3)$ & $65.00 \pm 5.03(3)^{* *}$ \\
TRI $(\mathrm{mg} / \mathrm{dl})$ & $55.00 \pm 3.22(3)$ & $16.07 \pm 0.93(3)^{*}$ \\
VLDL $(\mathrm{mg} / \mathrm{dl})$ & $11.00 \pm 0.64(3)$ & $4.67(3)^{*}$ \\
\hline
\end{tabular}

Los valores se expresan como media \pm SEM (número de animales entre paréntesis)

Análisis estadístico realizado mediante la prueba $t$ de Student para datos no apareados. ${ }^{* *} p<0.01, *$ $p<0.05$

Leucocitos (WBC), hemoglobina (HGB), eritrocitos (RBC), hematocritos (HCT) volumen corpuscular medio (MCV), hemoglobina corpuscular media $(\mathrm{MCH})$, concentración de hemoglobina corpuscular media (MCHC), tamaño y dispersión de los eritrocitos (RDW-CV, RDW-SD), conteo de plaquetas (PLT), volumen plaquetario medio (MPV), índice de distribución de plaquetas (PDW), porcentaje de procalcitonina $(P C T)$, concentraciones de glucosa $(\mathrm{GLU})$, colesterol (COL), triglicéridos (TRI) y lipoproteínas de muy baja densidad (VLDL)

tránsito intestinal más lento, lo que podría producir una mayor absorción de agua a nivel intestinal y, por ende, mayor excreción (Qian, 2018).

La cantidad de proteínas de la dieta control fue superior a la concentración de proteínas de la dieta EWP; sin embargo, las excreciones urinarias de proteínas son similares entre ambos grupos experimentales, datos que concuerdan con la literatura estu- diada (Shevock et al., 1993. Gautier et al., 2014). Así mismo, se evidencia en ambos grupos una concentración plasmática de proteínas en el rango de normalidad (Caridad et al., 2011) (Cuadro 7).

\section{Parámetros Biológicos}

En el análisis bioquímico de sangre se observó una diferencia significativa en el volumen corpuscular medio (MCV), con un 
Cuadro 9. Ingesta de minerales en ratas Sprague Dawley alimentadas con dieta control y dieta EWP (semi-purificada con base de clara de huevo)

\begin{tabular}{ccc}
\hline \multirow{2}{*}{ Mineral } & \multicolumn{2}{c}{ Ingesta $(\mathrm{mg} / 24 \mathrm{~h})$} \\
\cline { 2 - 3 } & Control $(\mathrm{n}=13)$ & $\mathrm{EWP}(\mathrm{n}=11)$ \\
$\mathrm{Al}$ & $3.01 \pm 0.06$ & $1.62 \pm 0.06 * * *$ \\
$\mathrm{Ba}$ & $0.37 \pm 0.01$ & $0.09 \pm 0.003 * * *$ \\
$\mathrm{Fe}$ & $15.01 \pm 0.44$ & $6.56 \pm 0.24 * * *$ \\
$\mathrm{Cu}$ & $1.14 \pm 0.02$ & $0.32 \pm 0.012 * * *$ \\
$\mathrm{Mn}$ & $1.98 \pm 0.04$ & $0.94 \pm 0.035^{* * *}$ \\
$\mathrm{Sr}$ & $0.68 \pm 0.01$ & $0.36 \pm 0.012 * * *$ \\
$\mathrm{Zn}$ & $13.3 \pm 0.26$ & $1.38 \pm 0.051 * * *$ \\
$\mathrm{Ca}$ & $102.7 \pm 2.01$ & $68.09 \pm 2.51 * * *$ \\
$\mathrm{~K}$ & $136.6 \pm 2.67$ & $97.06 \pm 3.58 * * *$ \\
$\mathrm{Mg}$ & $22.87 \pm 0.45$ & $13.63 \pm 0.50 * * *$ \\
$\mathrm{Na}$ & $25.54 \pm 0.51$ & $48.05 \pm 1.77 * * *$ \\
$\mathrm{P}$ & $61.66 \pm 1.21$ & $39.71 \pm 1.46 * * *$ \\
\hline
\end{tabular}

Los valores se expresan como media \pm SEM

Análisis estadístico realizado mediante la prueba t de Student para datos no apareados.

$* * * \mathrm{p}<0.001$

Cuadro 10. Concentración plasmática de minerales en ratas Sprague Dawley alimentadas con dieta control y dieta EWP (semi-purificada con base de clara de huevo)

\begin{tabular}{ccc}
\hline Mineral & Control & EWP \\
\hline $\mathrm{Cu}(\mu \mathrm{g} / \mathrm{m})$ & $1.12 \pm 0.13(6)$ & $1.19 \pm 0.08(3)$ \\
$\mathrm{Zn}(\mu \mathrm{g} / \mathrm{ml})$ & $1.17 \pm 0.21(7)$ & $0.70 \pm 0.16(3)^{* *}$ \\
$\mathrm{Ca}(\mathrm{mmol} / \mathrm{l})$ & $2.05 \pm 0.40(6)$ & $1.55 \pm 0.26(3)$ \\
$\mathrm{K}(\mathrm{mmol} / \mathrm{l})$ & $4.74 \pm 0.44(7)$ & $4.36 \pm 0.53(3)$ \\
$\mathrm{Mg}(\mathrm{mmol} / \mathrm{l})$ & $1.23 \pm 1.77(6)$ & $0.72 \pm 0.18(3)^{*}$ \\
$\mathrm{Na}(\mathrm{mmol} / \mathrm{l})$ & $119.83 \pm 1.35(7)$ & $103.29 \pm 2.72(3)$ \\
$\mathrm{P}(\mathrm{mmol} / \mathrm{l})$ & $4.57 \pm 0.31(6)$ & $3.52 \pm 0.32(3)^{*}$ \\
\hline
\end{tabular}

Los valores se expresan como media \pm SEM (Número de animales entre paréntesis) Análisis estadístico realizado mediante la prueba $t$ de Student para datos no apareados $* * * p<0.001$

valor de $+3.3 \pm 0.62 \mathrm{fl}$. También, se observó una diferencia significativa de $+27.33 \pm 2.43$ $\mathrm{mg} / \mathrm{dl}$ en COL, $+25.33 \pm 1.45 \mathrm{mg} / \mathrm{dl}$ en TRI y una diferencia de $+5.07 \pm 0.29 \%$ en el nivel de VLDL en ratas alimentadas con dieta EWP con respecto a ratas alimentadas con dieta control (Cuadro 8). El ligero aumento en COL, TRI y VLDL puede deberse al con- 
tenido de azúcares simples por añadidura de papelón en la dieta EWP. Sin embargo, los valores presentados por ambos grupos se encuentran dentro de los rangos «normales» para ratas (Caridad et al., 2011).

Se encontró una mayor ingesta de minerales en ratas alimentadas con EWP en comparación con el control (Cuadro 9), debido posiblebmente a que la dieta EWP cumple con los requerimientos establecidos por AIN (Reeves et al., 1993) (Cuadro 3). Al evaluar la concentración plasmática de minerales se evidencian rangos normales en ambas dietas (Owen y Hazelrig, 1968; Wilkins et al., 1972; Bansal, 1990; Lillie et al., 1996), a excepción de Zn, Mg y P. Esto se debe a que la concentración de dichos minerales en la dieta EWP son más cercanos a los requerimientos de AIN que en la dieta control (Cuadro 10).

\section{Conclusiones}

- La dieta EWP es una alternativa alimentaria controlada dirigida a ratas y ratones tal como lo sugieren las directrices del AIN, cubriendo los requerimientos de energía, macro y micronutrientes de las ratas Sprague Dawley, sin afecciones ni padecimientos subyacentes.

- Los parámetros clínico-metabólicos y biológicos demuestran que la dieta EWP permite el desarrollo óptimo y sano de las ratas Sprague Dawley.

- La dieta EWP, por ser una dieta pulverizada, permite al investigador manipular cada uno de sus ingredientes en función de los requerimientos experimentales, pudiendo elaborar dietas específicas para evaluar diferentes parámetros de índole nutricional.

\section{Agradecimientos}

Se agradece a la Dirección del Instituto de Tecnología de Alimentos (ICTA-UCV) y al Instituto Nacional de Nutrición (INN) por el apoyo logístico en la elaboración y análisis proximal de la dieta EWP, y al Instituto Nacional de Investigación Agronómica (INRA), Centro de investigación de Clermont-Ferrand-Theix por la donación de vitaminas y minerales para su suplementación.

\section{Literatura Citada}

1. Bansal VK. 1990. Serum inorganic phosphorus. In: Walker HK., Clinical Methods: the history, physical, and laboratory examinations. Texas, USA: Butterworths Publishers.

2. Bieri JG, Stoewsand GS, Briggs GM, Phillips RW, Woodard JC, Knapka JJ. 1977. Report of the American Institute of Nutrition ad hoc Committee on Standards for Nutritional Studies. J Nutr 107: 13401348. doi: $10.1093 / \mathrm{jn} / 107.7 .1340$

3. Blumenreich MS. 1990. The white blood cell and differential count. In: Clinical methods: the history, physical, and laboratory examinations. Texas, USA: Butterworths Publishers.

4. Bradford M. 1976. A rapid and sensitive method for the quantification of microgram quantities of protein utilizing the principle of protein-dye dinding. Anal Biochem 72: 248-254. doi: 10.1006/abio.1976.9999

5. Caridad LGA, Blanco D, Peña A, Ronda M, González BO, Arteaga ME, Bada AM, et al. 2011. Valores hematológicos y bioquímicos de las ratas Sprague Dawley producidas en CENPALAB, Cenp: SPRD. REDVET 12(11). [Internet]. Disponible en: https:// www.redalyc.org/articulo.oa? $\mathrm{id}=63622049001$

6. Cheuvront SN, Fraser CG, Kenefick RW, Ely BR, Sawka MN. 2011. Reference change values for monitoring dehydration. Clin Chem Lab Med 49:1033-1037. doi: 10.1515/CCLM. 2011.170

7. Christians JK, Lennie KI, Wild LK, Garcha R. 2019. Effects of high-fat diets on fetal growth in rodents: a systematic 
review. Reprod Biol Endocrinol 17(1): 39. doi: 10.1186/s12958-019-0482-y

8. Endo N, Rahayu LP, Arawaka T, Tanaka T. 2016. Video tracking analysis of behavioral patterns during estrus in goats. J Reprod Dev 62: 115-119. doi: 10.1262/jrd.2015-118

9. Gautier JC, Gury T, Gufroy M, KhanMalek R, Hoffman D, Pettit S, Harpur E. 2014. Normal ranges and variability of novel urinary renal biomarkers in SpragueDawley rats: comparison of constitutive values between males and females and across assay platforms. Toxic Pathol 42: 1092-1104. doi: 10.1177/0192623313520352

10.Horwitz, W, Latimer GW. 2005. Official methods of analysis of AOAC International. USA: AOAC linternational.

11. Kind KL, Moore VM, Davies MJ. 2006. Diet around conception and during pregnancy - effects on fetal and neonatal outcomes. Reproduc BioMed Online 12: 532-541. doi: 10.1016/s1472-6483 (10)61178-9

12.Levin BE, Keesey RE. 1998. Defense of differfing body weight set points in dietinduced obese and resistant rats. In Yosten GL. Regulatory and integrative physiology. Rockville, USA: The American Physiological Society. pp R412-R419.

13. Lillie LE, Temple NJ, Florence LZ. 1996. Reference values for young normal Sprague-Dawley rats: weight gain, hematology and clinical chemistry. Hum Exp Toxicol 15: 612-616. doi: 10.1177/096032719601500802

14. Martus W, Kim D, Garvin JL, Beierwaltes WH. 2005. Commercial rodent diets contain more sodium than rats need. Am J Physiol Renal Physiol 288: F428-F431. doi: 10.1152/ajprenal. 00310.2004

15. Morton DB, Griffiths PHM. 1985. Guidelines on the recognition of pain, distress and discomfort in experimental animals and an hypothesis for assessment. Vet Rec 116: 431-436. doi: 10.1136/vr.116.16.431
16. Naim M, Brand JG, Christensen CM, Kare MR, Van Buren S. 1986. Preference of rats for food flavors and texture in nutritionally controlled semipurified diets. Physiol Behav 37: 15-21. doi: 10.1016/0031-9384(86)90377-x

17. Olivares E, Herrera F, Aguiar G, Peña E, Ramos M, Méndez C. 2016. Comparación de la nutrición mineral de los helechos Cyathea aurea, $C$. delgadii, Dicranopteris flexuosa y Pteridium arachnoideum en la Gran Sabana, Venezuela. Interciencia 42: 273-283.

18. Owen CA Jr, Hazelrig JB. 1968. Copper deficiency and copper toxicity in the rat. Am J Physiol 215: 334-338. doi: 10.1152/ajplegacy.1968.215.2.334

19. Qian Q. 2018. Dietary influence on body fluid acid-base and volume balance: the deleterious «norm» furthers and cloaks subclinical pathophysiology. Nutrients 10: 778. doi: 10.3390/ nu10060778

20. Ramirez I, Friedman MI. 1990. Dietary hyperphagia in rats: role of fat, carbohydrate, and energy content. Physiol Behav 47: 1157-1163. doi: 10.1016/0031-9384(90)90367-d

21. Reeves PG, Rossow KL, Lindlauf J. 1993a. Development and testing of the AIN-93 purified diets for rodents: results on growth, kidney calcification and bone mineralization in rats and mice. J Nutr 123: 1923-1931. doi: 10.1093/ $\mathrm{jn} / 123.11 .1923$

22. Reeves PG, Nielsen FH, Fahey Jr $G C .1993 b$. AIN-93 purified diets for laboratory rodents: final report of the American Institute of Nutrition ad hoc writing commitee on the reformulation of the AIN-76A rodent diet. J Nutr 123: 1939-1951. doi: 10.1093/jn/ 123.11.1939 
23. Rieble SK, Davy BM. 2013. The hydration equation: update on water balance and cognitive performance. ACSMs Health Fit J 17: 21-28. doi: 10.1249/FIT.0b013e3182a9570f

24. Rondón LJ, Marcano E, Rodríguez F, del Castillo JR. 2014. Blood pressure, magnesium and other mineral balance in two rat models of saltsensitive, induced hypertension: effects of a non-peptide angiotensin II receptor type 1 antagonist. Magnes Res 27: 113130. doi: $10.1684 / \mathrm{mrh} .2014 .0368$

25. Roumelioti ME, Glew RH, Khitan ZJ, Rondon-Berrios H, Argyropoulos CP, Malhotra D, Raj DS, et al. 2018. Fluid balance concepts in medicine: principles and practice. World J Nephrol 7: 1-28. doi: 10.5527/ wjn.v7.i1.1

26. Saínz MB. 2005. Balance hidromineral (BHM). Rev Cubana Cir 44(4). [Internet]. Disponible en: https:// docplayer.es/3812006-Balancehidromineral-bhm.html
27. Semon BA, Leung PMB, Rogers QR, Gietzen DW. 1987. Effect of type of protein on food intake of rats fed a high protein diets. Physiol Behav 41: 451-458. doi: 10.1016/0031-9384(87)90080-1

28. Shevock PN, Khan SR, Hackett RL. 1993. Urinary chemistry of the normal Sprague-Dawley rat. Urol Res 21: 309312. doi: 10.1007/BF00296826

29. Sotocinal SG, Sorge RE, Zaloum A, Tuttle AH, Martin LJ, Wieskopf JS, et al. 2011. The Rat Grimace Scale: a partially automated method for quantifying pain in the laboratory rat via facial expressions. Mol Pain 7(55). doi: 10.1186/1744-8069-7-55

30. Wilkins PJ, Grey PC, Dreos IE. 1972. Plasma zinc as an indicator of zinc status in rats. Br J Nutr 27: 113-120. doi: 10.1079/bjn19720075

31. Windsor DL, Denton MB. 1978. Evaluation of inductively coupled plasma optical emission spectrometry as a method for the elemental analysis of organic compounds. Appl Spectrosc 32: 366-371. doi: 10.1366/000370278774331134 